\title{
Accumulation of factors influencing respiratory illness in members of a national birth cohort and their offspring
}

\author{
S L Mann, M E J Wadsworth, J R T Colley
}

\begin{abstract}
Study objective-The aim was to investigate predictors of childhood lower respiratory tract illness in two generations, and predictors of adult lower respiratory disorders in the first generation.

Design-Data on respiratory health and environmental factors from a national birth cohort study were examined from birth to 36 years. Data were also collected on the parents of the subjects and on the subjects' first born offspring from birth to eight years. Main outcome measures were: reports of lower respiratory tract illness before 2 years; lower respiratory tract illness of a week or more between age 20 and 36 years; regular phlegm production at 25 and 36 years; reports of wheeze or asthma at age 36 years; peak expiratory flow rate (PEFR) at age 36 years measured by nurses during home visits; and mothers' reports of lower respiratory illness in first born offspring before 2 years.
\end{abstract}

Subjects-Subjects were a sample of 5362 single, legitimate births taken from all those occurring in England, Wales, and Scotland in one week in 1946, and studied regularly from birth to age 43 years. Data on the subjects' parents and on their 1676 first offspring born while they were aged 19-25 years were also collected.

Main results-Lower respiratory tract illness before 2 years fell from $25 \%$ in the population born in 1946 to $13 \%$ in their first born offspring. In those born in 1946, poor home environment, parental bronchitis, and atmospheric pollution were the best predictors of lower respiratory illness before 2 years, and these three factors and childhood lower respiratory illness and later smoking were the best predictors of adult lower respiratory tract problems. Risk factors for lower respiratory illness in the offspring were manual social class, parental and grandparental lower respiratory disease, and parental smoking.

Conclusions-Risks for adult lower respiratory problems accumulated in childhood through illness, poor social circumstances, and atmospheric pollution. Smoking exacerbated early life risks and was an independent risk factor. In the offspring generation, parental smoking was a risk factor for early life chest illness, together with parental illness and low social class. Reduction of prevalence in the offspring generation was probably accounted for by improvement in home circum- stances, reduced atmospheric pollution, and lower rates of parental lower respiratory illness, but current rates of smoking seem likely to prevent much further reduction in early life lower respiratory illness, and thus in this aspect of risk for subsequent adult lower respiratory problems. The accumulation of risk in childhood and adolescence for later adult problems implies a long time scale for the reduction of adult lower respiratory disorders.

Since Reid and others drew attention to the presumptive link between childhood lower respiratory tract illness and later adult chronic bronchitis $^{1-3}$ many studies have shown the association of early life lower respiratory illness with later increased risk of lower respiratory problems in childhood, ${ }^{4-7}$ in adolescence or young adult life, ${ }^{8-11}$ and in older adults. ${ }^{11-14}$ Migrant studies offer some confirmation of the childhood risks. ${ }^{15}$

Evidence for the risk of lower respiratory illness during childhood from poor social and home circumstances, ${ }^{16-19}$ parental smoking, ${ }^{20-22}$ and exposure to high levels of atmospheric smoke pollution ${ }^{7-9} 1723$ is strong. Adult bronchitis has been predicted both by childhood lower respiratory illness and by cigarette smoking. ${ }^{17}$ Protective effects against childhood lower respiratory tract disease have been found in breast feeding. ${ }^{24}$

Hypotheses put forward to explain the long term association of childhood lower respiratory illness with adult bronchitis include the suggestions that bronchopulmonary insults associated with early life chest illness may be the source of vulnerability to later lower respiratory tract disease, ${ }^{111416}$ that the continued association is the result of asthma ${ }^{10}$ or of poor social conditions in childhood, ${ }^{15-19}$ or of other and as yet unidentified host factors. ${ }^{6}$

This paper compares the prevalence of lower respiratory illness in three generations of the same families, the middle one of which comprised members of a national birth cohort study, and examines the relationships within and between generations of risk factors for lower respiratory problems in childhood and in adult life during a period of falling prevalence and changing risk factors.

\section{Methods}

The Medical Research Council (MRC) National Survey of Health and Development is a follow up study of all single, legitimate births to wives of non-manual and agricultural workers, and one in 
four of all single, legitimate births to wives of manual workers in England, Wales, or Scotland in the week 3rd-9th March, 1946. A weighting procedure compensates for the stratification by fathers' social class at the time of the study member's birth by multiplying the results of those in the sampled classes (one in four of manual workers' children) by the denominator value of the sampling fraction.

\section{INFORMATION ON STUDY MEMBERS}

Information was collected at intervals of two years or less in childhood and adolescence, and at intervals of approximately five years in adult life, so far to age 43 years. At each contact a wide range of age appropriate data was collected on medical, social, psychological, educational, and occupational topics. ${ }^{25}$ At age 36 years, 56 research nurses successfully interviewed and measured 3322 study members in their homes, $86 \%$ of the 3875 alive $(6 \%$ of the original sample had died by this age), resident in England, Wales, or Scotland $(12.8 \%$ lived abroad) and still participant in the study $(10.0 \%$ had refused).

Five respiratory health indicators were used, one in childhood and four in adult life. Data on lower respiratory illness between birth and 15 years (bronchitis, pneumonia, bronchopneumonia, bronchiectasis, bronchiolitis) were collected by health visitors from mothers and from hospital and clinic records, and by school doctors and nurses at examinations of the study children undertaken at ages $6,7,11$, and 15 years; these data were combined to make one variable describing reports of these illnesses before two years, and another of reports of these illnesses after two years. All reports of hospital admissions for these and all other illnesses were checked with hospitals. Information on asthma was collected in the same way, but no specific question about this condition was asked until the medical examination at age 6 years. The MRC questions on chronic cough ${ }^{26}$ were asked at ages 25,31 , and 36 years, and on phlegm at ages 25 and 36 years. Reports of lower respiratory illness of at least one week duration were collected at 20,25, 31, and 36 years, reports of wheezing or experience of asthma were recorded at age 36 years, and all were checked wherever appropriate with hospital inpatient notes. At 36 years peak flow rates (PEFR) were measured by research nurses using the mini Wright peak flow meter.

Table I Percentage prevalence of chest illness by two years adjusted for effect of associated factors $(n=2133)$

\begin{tabular}{|c|c|c|c|c|c|}
\hline $\begin{array}{l}\text { Associated } \\
\text { factors }\end{array}$ & & $\begin{array}{l}\text { Adjusted } \\
\text { prevalence }(\%) \\
\text { chest illness } \\
\text { by two years }\end{array}$ & $\begin{array}{l}\text { Test } \\
\text { statistic }^{\mathrm{a}}\end{array}$ & $d f$ & $p$ \\
\hline Maternal care & $\begin{array}{l}\text { good } \\
\text { poor }\end{array}$ & $\begin{array}{l}25^{\circ} \\
29^{\circ}\end{array}$ & $3 \cdot 17$ & 1 & NS \\
\hline Amenities lacking & $\begin{array}{l}\text { none } \\
\text { one or more }\end{array}$ & $\begin{array}{l}26^{\circ} \circ \\
28 \%\end{array}$ & $0 \cdot 756$ & 1 & NS \\
\hline $\begin{array}{l}\text { Atmospheric pollution } \\
\text { exposure }\end{array}$ & $\begin{array}{l}\text { low } \\
\text { high }\end{array}$ & $\begin{array}{l}23{ }^{\circ} \\
31^{\circ} \%\end{array}$ & $16 \cdot 525$ & 1 & $<0.001$ \\
\hline Parental bronchitis & $\begin{array}{l}\text { no } \\
\text { yes }\end{array}$ & $\begin{array}{l}23{ }^{\circ} \\
31^{\circ}\end{array}$ & $10 \cdot 187$ & 1 & $<0.01$ \\
\hline $\begin{array}{l}\text { Social class } \\
\text { of father }\end{array}$ & $\begin{array}{l}\text { non-manual } \\
\text { manual }\end{array}$ & $\begin{array}{l}23^{\circ} \mathrm{o} \\
31^{\circ} \%\end{array}$ & $11 \cdot 642$ & 1 & $<0.001$ \\
\hline $\begin{array}{l}\text { Crowding at two years } \\
\text { (persons per room) }\end{array}$ & $\begin{array}{l}<1 / \text { room } \\
>1 / \text { room }\end{array}$ & $\begin{array}{l}24^{\circ}{ }^{\circ} \\
29{ }^{\circ}\end{array}$ & 5.633 & 1 & $<0.05$ \\
\hline
\end{tabular}

${ }^{\mathrm{a}}$ Likelihood ratio test statistic
Exposure to atmospheric pollution in childhood was assessed eight times between birth and 11 years using information on local domestic coal consumption supplied by the Warren Spring Laboratory. ${ }^{7}$ Adult exposure to sulphur dioxide pollution was assessed at 26 years, also using Warren Spring Laboratory information.

Smoking habits were reported at interviews with a research nurse at age 36 years, and by postal questionnaire at ages 20,25 , and 31 years.

Measures of physical development comprise birth weight, collected by health visitors at age eight weeks, a medical assessment of state of physical maturity at 15 years, and a measure of height taken by a nurse at 36 years.

Mother's care of the study child was assessed by health visitors, when children were aged 2,4 , and 6 years, and data on breast feeding, availability of three amenities (hot running water and sole use of kitchen and bathroom), and crowding at age 2 years were collected at the same home visit.

\section{INFORMATION ON STUDY MEMBERS' PARENTS}

Information on lower respiratory illness in parents was obtained at interviews with study members at age 36 years.

INFORMATION ON OFFSPRING OF STUDY MEMBERS Information on lower respiratory problems and home circumstances of first born offspring of study members was collected from their mothers at home visits when these children were aged four and eight years. The sample of offspring comprises all first born children born when study members were aged 19-25 years; 1684 were visited at age four years and 1676 at eight years. ${ }^{27} 28$

\section{Results}

STUDY MEMBERS

Lower respiratory illness in early childhood (by two years)

Bronchitis, pneumonia, bronchopneumonia, bronchiectasis, or bronchiolitis (collectively referred to here as lower respiratory illness) were experienced by $25^{\circ}$ of the study group before the age of two years (1946-48) (and by $28^{\circ}{ }_{0}$ of the weighted population); asthma was reported in one study member by this age, and this child was also reported to have had bronchitis. There was no significant sex difference. The risk of lower respiratory illness before two years was significantly raised by exposure to high levels of atmospheric pollution, parental bronchitis, a crowded household, father in manual employment, poor maternal care, and lack of household amenities (all with $\mathrm{p}<0.001$ ). Low birth weight $(<2500 \mathrm{~g})$, breast feeding, and parental asthma were not associated with these reported childhood lower respiratory illnesses.

After adjusting for all the statistically significant factors listed above, crowded conditions at age two years, history of parental bronchitis, father's social class, and exposure to atmospheric pollution remained independently associated with lower respiratory illness before two years (table I).

Relationship of adult respiratory problems with earlier life risk factors

At 36 years information was collected on the following indicators of lower respiratory 
problems: the experience of bronchitis, pneumonia, or other chest illness since age 20 years $(30 \%$ of the study population); positive response to the MRC questions ${ }^{26}$ on regular winter cough $(14 \%)$ and phlegm production since age 25 years $(8 \%)$; any adult reports of wheezing or whistling sounds in the chest or asthma $(24 \%)$ and a measurement of peak expiratory flow rate (PEFR) at 36 years. The rate of reporting one or more of the five symptoms or illnesses was $30.4 \%$ of the population studied at age 36 years. The mean adjusted PEFR value for males was 554.5 litres/min (SE 1.97) and for females 411.9 litres $\min$ (SE 1.67). The accumulation of risk of adult lower respiratory problems between the ages of two and 36 years was examined, in order to investigate whether the long term association was best predicted by lower respiratory illness before two years and its associated risk factors, or by risk factors experienced later.

Evidence for the predictive value of childhood lower respiratory illness for later adult lower respiratory problems was strong: $33 \%$ of those who had lower respiratory illness between 20 and 36 years also had lower respiratory illness before two years $(p<0.001)$. Adult reports of phlegm production, asthma or wheezing, and lower respiratory illness were also significantly greater among those who had experienced early childhood lower respiratory illness (table II), but cough at 25 and 36 years was not, and was therefore not considered in further analyses. Lower respiratory illness in childhood was associated with significantly increased risk of being in the lowest quartile of PEFR at 36 years (table II).

Those who experienced lower respiratory illness before two years accumulated further risk later on in childhood and adolescence. They were significantly more inclined to be still living in areas of relatively high atmospheric pollution, and to be smokers (table II). In analyses not shown, lower respiratory illness before two years was not significantly associated with change in social class in later childhood or in adult life, or with age at attainment of physical maturity, assessed by a school doctor.

Vulnerability to adult lower respiratory illness in the population of those who did not experience such disease before two years was significantly

Table II Prevalence of risk factors and adult illness by history of chest illness up to two years

\begin{tabular}{|c|c|c|c|c|}
\hline $\begin{array}{l}\text { Potential risk } \\
\text { factors }\end{array}$ & $\begin{array}{l}\text { No chest } \\
\text { illness } \\
\text { by two years }\end{array}$ & $\begin{array}{l}\text { One or more } \\
\text { chest } \\
\text { illnesses } \\
\text { by two years }\end{array}$ & $\chi^{2} / F^{\star}$ & $p$ \\
\hline \multirow{5}{*}{$\begin{array}{l}\text { Asthma (recorded at } 6,11,14 \text {, and } \\
36 \text { years })(n=179) \\
\text { Exposure to high atmospheric pollution } \\
\text { at } 2-11 \text { years }(n=1935) \\
\text { Late puberty }(n=1920) \\
\text { Current smoker at } 36 \text { years }(n=1040) \\
\text { Ex-smoker at } 36 \text { years }(n=1121) \\
\text { Never smoked at } 36 \text { years }(n=924) \\
\text { High exposure to } \mathrm{SO}_{2} \text { at } 26 \text { years } \\
(\mathrm{n}=581)\end{array}$} & $6 \%$ & $11 \%$ & $15 \cdot 31$ & $<0.001$ \\
\hline & \multirow{4}{*}{$\begin{array}{l}46 \% \\
54 \% \\
32 \% \\
37 \% \\
31 \% \\
15 \%\end{array}$} & \multirow{4}{*}{$\begin{array}{l}57 \% \\
52 \% \\
38 \% \\
35 \% \\
27 \% \\
18 \%\end{array}$} & $\begin{array}{c}35 \cdot 4 \\
1.31\end{array}$ & $\begin{array}{l}<0 \cdot 001 \\
\text { NS }\end{array}$ \\
\hline & & & \multirow{2}{*}{$7 \cdot 722$} & \multirow{2}{*}{$<0.05$} \\
\hline & & & & \\
\hline & & & $4 \cdot 27$ & $<0.05$ \\
\hline \multirow{3}{*}{$\begin{array}{l}\text { Cough (at } 25,31 \text {, and } 36 \text { years) }(\mathrm{n}=288) \\
\text { Phlegm (at } 25 \text { and } 36 \text { years) }(\mathrm{n}=180) \\
\text { Lower respiratory illness (at } 20,25,31, \\
\text { and } 36 \text { years) }(\mathrm{n}=691) \\
\text { Asthma or wheeze (at } 36 \text { years) }(\mathrm{n}=738)\end{array}$} & outcome indice & cators & & \multirow[b]{2}{*}{$\begin{array}{l}\text { NS } \\
<0.001\end{array}$} \\
\hline & $\begin{array}{r}13 \% \\
7 \%\end{array}$ & $\begin{array}{l}16 \% \\
12 \%\end{array}$ & $\begin{array}{r}1 \cdot 836 \\
17 \cdot 489\end{array}$ & \\
\hline & $\begin{array}{l}27 \% \\
22 \%\end{array}$ & $\begin{array}{l}38 \% \\
30 \%\end{array}$ & $\begin{array}{l}25.031 \\
21.652\end{array}$ & $\begin{array}{l}<0.001 \\
<0.001\end{array}$ \\
\hline \multicolumn{5}{|l|}{$\begin{array}{l}\text { Height and sex adjusted mean } \\
\text { PEFR litre/min (at } 36 \text { years): }\end{array}$} \\
\hline $\begin{array}{l}\text { males }(n=1522) \\
\text { females }(n=1524)\end{array}$ & $\begin{array}{l}556 \cdot 32 \\
413 \cdot 43\end{array}$ & $\begin{array}{l}547 \cdot 57 \\
\mathbf{4 0 4} \cdot 65\end{array}$ & $\begin{array}{l}3 \cdot 55^{\star} \\
4 \cdot 66^{\star}\end{array}$ & $\begin{array}{l}<0.06 \\
<0.05\end{array}$ \\
\hline
\end{tabular}

increased in those who had grown up in low social class families ( $12 \%$ attributable risk), had parents who had had chest illness ( $9 \%$ attributable risk), had experienced high atmospheric pollution $(7 \%$ attributable risk), or were cigarette smokers (37\% attributable risk). For those in this group from non-manual families of origin, smoking was the most powerful predictor of adult lower respiratory illness.

By 36 years $70 \%$ of the population were either current cigarette smokers $(34 \%)$ or ex-cigarette smokers $(36 \%)$. There were no significant differences in the relationship of adult lower respiratory illness, phlegm or wheezing, or asthma with current and previous smoking. However PEFR was lower among current smokers in both males $(p<0.001)$ and females $(p<0.01)$. In adults who had been free from both early life lower respiratory illness and exposure to childhood environmental risk factors, cigarette smoking was associated with raised risk of adult lower respiratory illness: $22 \%$ versus $11 \%$ of non-smokers. Among non-smokers who were free from early life lower respiratory illness and who came from poor childhood environments, $18 \%$ had adult lower respiratory illness compared with $30 \%$ of smokers $(\mathrm{p}<0.05)$.

Those from low social class families of origin who lived during childhood in overcrowded circumstances with few amenities had a reduced risk of adult lower respiratory disease if they had also been breast fed $(p<0 \cdot 01),{ }^{13}$ but no greater likelihood than others of cigarette smoking in adolescence or later life.

Thus the importance of childhood chest illness as a risk factor for adult lower respiratory problems was significant, but not uniquely necessary. Of all those who at 36 years reported one or more of the adult symptoms or illnesses, $29 \cdot 6 \%$ had also had childhood lower respiratory illness. Only $13.7 \%$ of those with adult lower respiratory problems had not had childhood illness and had not been smokers (table III).

Table III Summary of the association of adult early life lower respiratory problems and their relationship with smoking at 36 years

\begin{tabular}{|c|c|c|c|c|c|}
\hline \multirow[b]{2}{*}{$\begin{array}{l}\text { Adult lower } \\
\text { respiratory } \\
\text { problems by } \\
36 \text { years }\end{array}$} & \multirow[b]{2}{*}{$\begin{array}{l}\text { Lower } \\
\text { respiratory } \\
\text { problems } \\
\text { by } 2 \text { years }\end{array}$} & \multicolumn{3}{|c|}{ Smoking at 36 years } & \multirow[b]{2}{*}{$\begin{array}{l}\text { Total } \\
(=100 \%)\end{array}$} \\
\hline & & $\begin{array}{l}\text { Current } \\
\text { smoker } \\
(\%)\end{array}$ & $\begin{array}{l}\text { Ex } \\
\text { smoker } \\
(\%)\end{array}$ & $\begin{array}{l}\text { Never } \\
\text { smoked } \\
(\%)\end{array}$ & \\
\hline \multirow[t]{2}{*}{ None } & \multirow{2}{*}{$\begin{array}{c}\text { None } \\
\text { One or } \\
\text { more }\end{array}$} & $23 \cdot 7$ & $40 \cdot 6$ & $35 \cdot 7$ & 1601 \\
\hline & & $27 \cdot 4$ & $38 \cdot 1$ & $34 \cdot 5$ & 449 \\
\hline \multirow[t]{2}{*}{ One or more } & \multirow{2}{*}{$\begin{array}{l}\text { None } \\
\text { One or } \\
\text { more }\end{array}$} & $53 \cdot 1$ & $28 \cdot 0$ & 18.9 & 631 \\
\hline & & 53.6 & 29.8 & 16.6 & 265 \\
\hline
\end{tabular}

Relationship of adult respiratory problems with all childhood and later risk factors

Factors associated with the risk of early childhood lower respiratory illness, and with risk accumulated in the intervening years, were examined in order to investigate their relative predictive value for adult lower respiratory problems.

Logistic regression methods were used to investigate the relationship of the three dichotomous outcome variables, phlegm production, wheeze and/or asthma, and adult chest illness, with their statistically significant risk 
factors. The association of the fourth adult variable, PEFR, with its risk factors was investigated by analysis of variance. Since some of the variation in lung function is dependent on height, the analyses of PEFR included an adjustment for height at age 36 years, and sex was included in every model as a dummy variable since mean PEFR was significantly greater in men than in women. Associations between outcome variables and each risk factor were explored, after allowing for other factors.

Regression analyses of adult lower respiratory problems and childhood lower respiratory illness under two years, with its associated risk factors (under two years), showed that only parental bronchitis was independently and significantly associated with all four adult outcome variables after the effects of all other variables had been taken into account (table IV). Lower respiratory illness under two years was also associated with adult phlegm production, wheeze or asthma, and lower respiratory tract illness, but low social class in childhood was associated only with low PEFR. Crowding was associated with all outcomes except adult lower respiratory illness. Atmospheric pollution before two years, breast feeding, and lack of amenities were no longer associated with any of the four adult outcome variables.

Further regression was carried out to examine the association of the four adult outcome variables with possible sources of risk occurring after two years. Cigarette smoking was significantly related to all adult outcome measures (table V). Exposure to high levels of atmospheric pollution between ages two and 11 years was also associated with significantly greater risk of adult lower respiratory illness and wheeze or asthma, but there was no association of adult lower respiratory problems with exposure to high $\mathrm{SO}_{2}$ levels at 26 years.

The significant childhood and later risk factors were combined in one regression analysis (table VI). Parental bronchitis and own cigarette smoking were associated with all four adult outcome measures. Childhood lower respiratory illness remained significantly associated with adult lower respiratory illness, wheeze or asthma, and phlegm production. Living in an overcrowded household at age two years was associated with phlegm, wheeze or asthma, and a decrease in PEFR. History of asthma was strongly associated with phlegm, adult lower respiratory illness, and decreased PEFR. Exposure to

Table IV Regression analysis of four indices of adult respiratory health with early childhood risk factors

\begin{tabular}{|c|c|c|c|c|c|c|c|c|c|c|c|c|c|}
\hline & & \multicolumn{3}{|c|}{$\begin{array}{l}\text { Phlegm at } 25 \\
\text { and } 36 \text { years } \\
(n=1613)\end{array}$} & \multicolumn{3}{|c|}{$\begin{array}{l}\text { Adult lower } \\
\text { respiratory illness } \\
(20-36 \text { years }) \\
(n=1593)\end{array}$} & \multicolumn{3}{|c|}{$\begin{array}{l}\text { Wheeze or } \\
\text { asthma (36 years) } \\
(n=2139)\end{array}$} & \multicolumn{3}{|c|}{$\begin{array}{l}\text { Mean height } \\
\text { adjusted PEFR } \\
\text { at } 36 \text { years } \\
(n=2107) \\
\end{array}$} \\
\hline & & $\%$ & $\begin{array}{l}\text { Test } \\
\text { statistic }\end{array}$ & $p$ & $\%$ & $\begin{array}{l}\text { Test } \\
\text { statistic } \\
\end{array}$ & $p$ & $\%$ & $\begin{array}{l}\text { Test } \\
\text { statistic }\end{array}$ & $p$ & $\begin{array}{l}\text { Mean } \\
\text { PEFR }\end{array}$ & $\begin{array}{l}\text { Test } \\
\text { statistic }\end{array}$ & $p$ \\
\hline \multirow{2}{*}{$\begin{array}{l}\text { Atmospheric pollution } \\
\text { exposure to } 2 \text { years }\end{array}$} & Low & 9 & \multirow{2}{*}{$0 \cdot 37$} & \multirow{2}{*}{ NS } & 28 & \multirow{2}{*}{$2 \cdot 25$} & \multirow{2}{*}{ NS } & 27 & \multirow{2}{*}{$1 \cdot 49$} & \multirow{2}{*}{ NS } & $480 \cdot 8$ & \multirow{2}{*}{$3 \cdot 3$} & \multirow{2}{*}{ NS } \\
\hline & High & 8 & & & 31 & & & 30 & & & $475 \cdot 3$ & & \\
\hline \multirow{2}{*}{$\begin{array}{l}\text { Chest illness before } \\
2 \text { years }\end{array}$} & No & 7 & \multirow{2}{*}{$5 \cdot 13$} & \multirow{2}{*}{$<0.05$} & 26 & \multirow{2}{*}{$8 \cdot 59$} & \multirow{2}{*}{$<0.01$} & 25 & \multirow{2}{*}{$8 \cdot 68$} & \multirow{2}{*}{$<0.01$} & $479 \cdot 5$ & \multirow[b]{2}{*}{$0 \cdot 69$} & \multirow[b]{2}{*}{ NS } \\
\hline & Yes & 11 & & & 34 & & & 32 & & & $476 \cdot 6$ & & \\
\hline \multirow{2}{*}{$\begin{array}{l}\text { History of parental } \\
\text { bronchitis }\end{array}$} & No & 7 & \multirow{2}{*}{6.9} & \multirow{2}{*}{$<0.05$} & 26 & \multirow{2}{*}{8.06} & \multirow{2}{*}{$<0.01$} & 24 & \multirow{2}{*}{$17 \cdot 76$} & \multirow{2}{*}{$<0.001$} & $483 \cdot 7$ & \multirow{2}{*}{$10 \cdot 14$} & \multirow{2}{*}{$<0.01$} \\
\hline & Yes & 11 & & & 34 & & & 34 & & & $472 \cdot 3$ & & \\
\hline \multirow{2}{*}{$\begin{array}{l}\text { Social class } \\
\text { in childhood }\end{array}$} & Non-manual & 8 & \multirow{2}{*}{1.45} & \multirow{2}{*}{ NS } & 28 & & & 28 & & & $481 \cdot 8$ & & \\
\hline & Manual & 10 & & & 31 & $1 \cdot 08$ & NS & 29 & $0 \cdot 29$ & NS & $474 \cdot 3$ & 4.96 & $<0.05$ \\
\hline Crowding (persons per & $<1$ & 7 & & & 27 & & & 25 & & & $483 \cdot 8$ & & \\
\hline 100in) al age 2 years & $>1$ & 11 & 4.09 & $20.0\rangle$ & 32 & $2 \cdot 36$ & NS & 33 & 13.73 & $<0.001$ & $472 \cdot 3$ & $11 \cdot 7$ & $<0.001$ \\
\hline Amenities & None & 9 & & & 31 & & & 29 & & & $479 \cdot 2$ & & \\
\hline $\begin{array}{l}\text { lacking at } \\
\text { age } 2 \text { years }\end{array}$ & One or more & 8 & 0.08 & NS & 28 & 0.00 & NS & 28 & 0.12 & NS & $476 \cdot 8$ & 0.51 & NS \\
\hline Breastfed & Yes & 8 & & & 27 & & & 28 & & & $481 \cdot 2$ & & \\
\hline & No & 9 & 0.18 & NS & 32 & $2 \cdot 76$ & NS & 29 & 0.02 & NS & $474 \cdot 9$ & $3 \cdot 1$ & NS \\
\hline
\end{tabular}

PEFR $=$ peak expiratory flow rate

Table $V$ Regression analysis of four indices of adult respiratory health with later childhood and early adult risk factors

\begin{tabular}{|c|c|c|c|c|c|c|c|c|c|c|c|c|c|}
\hline & & \multicolumn{3}{|c|}{$\begin{array}{l}\text { Phlegm at } 25 \\
\text { and } 36 \text { years } \\
(n=1653)\end{array}$} & \multicolumn{3}{|c|}{$\begin{array}{l}\text { Adult lower } \\
\text { respiratory illness } \\
(20-36 \text { years) } \\
(n=1656)\end{array}$} & \multicolumn{3}{|c|}{$\begin{array}{l}\text { Wheeze or } \\
\text { asthma (36 years) } \\
(n=2688)\end{array}$} & \multicolumn{3}{|c|}{$\begin{array}{l}\text { Mean height } \\
\text { adjusted PEFR } \\
\text { at } 36 \text { years } \\
(n=2035)\end{array}$} \\
\hline & & $\%$ & $\begin{array}{l}\text { Test } \\
\text { statistic }\end{array}$ & $p$ & $\%$ & $\begin{array}{l}\text { Test } \\
\text { statistic }\end{array}$ & $p$ & $o_{0}$ & $\begin{array}{l}\text { Test } \\
\text { statistic }\end{array}$ & $p$ & $\begin{array}{l}\text { Mean } \\
\text { PEFR }\end{array}$ & $\begin{array}{l}\text { Test } \\
\text { statistic }\end{array}$ & $p$ \\
\hline \multirow{2}{*}{$\begin{array}{l}\text { Exposure to sulphur } \\
\text { dioxide at } 26 \text { years }\end{array}$} & Low & 9 & \multirow{2}{*}{$2 \cdot 17$} & \multirow{2}{*}{ NS } & 35 & \multirow{2}{*}{0.804} & \multirow{2}{*}{ NS } & 21 & \multirow{2}{*}{$0 \cdot 609$} & \multirow{2}{*}{ NS } & $475 \cdot 5$ & \multirow{2}{*}{$2 \cdot 5$} & \multirow{2}{*}{ NS } \\
\hline & High & 12 & & & 39 & & & 19 & & & $468 \cdot 3$ & & \\
\hline Cigarette smoking & $\left.\begin{array}{c}\text { Never } \\
\text { Ex } \\
\text { Current }\end{array}\right\}$ & $\begin{array}{r}8 \\
14\end{array}$ & $10 \cdot 42$ & $<0.01$ & $\begin{array}{l}29 \\
45\end{array}$ & $26 \cdot 472$ & $<0.001$ & $\begin{array}{l}14 \\
28\end{array}$ & $66 \cdot 405$ & $<0.001$ & $\begin{array}{l}473 \cdot 8 \\
476 \cdot 6 \\
465 \cdot 6\end{array}$ & $4 \cdot 65$ & $<0.01$ \\
\hline \multirow{2}{*}{$\begin{array}{l}\text { Atmospheric pollution } \\
2-11 \text { years }\end{array}$} & Low & 10 & \multirow{2}{*}{$0 \cdot 16$} & \multirow{2}{*}{ NS } & 34 & \multirow{2}{*}{$5 \cdot 849$} & \multirow{2}{*}{$<0.05$} & 18 & \multirow{2}{*}{$5 \cdot 098$} & \multirow{2}{*}{$<0.05$} & $474 \cdot 9$ & \multirow{2}{*}{$3 \cdot 42$} & \multirow{2}{*}{ NS } \\
\hline & High & 11 & & & 40 & & & 22 & & & $468 \cdot 9$ & & \\
\hline \multirow[t]{2}{*}{ Asthma history } & No & 7 & \multirow{2}{*}{$8 \cdot 74$} & \multirow{2}{*}{$<0.01$} & 21 & \multirow[b]{2}{*}{$56 \cdot 689$} & \multirow[b]{2}{*}{$<0.001$} & & & & $486 \cdot 5$ & \multirow[b]{2}{*}{$12 \cdot 76$} & \multirow[b]{2}{*}{$<0.001$} \\
\hline & Yes & 16 & & & 56 & & & & & & $457 \cdot 3$ & & \\
\hline
\end{tabular}


Table VI Regression analysis of four indices of adult respiratory health with all significant risk factors

\begin{tabular}{|c|c|c|c|c|c|c|c|c|c|c|c|c|c|}
\hline & & \multicolumn{3}{|c|}{$\begin{array}{l}\text { Phlegm at } 25 \\
\text { and } 36 \text { years } \\
(n=1506)\end{array}$} & \multicolumn{3}{|c|}{$\begin{array}{l}\text { Adult lower } \\
\text { respiratory illness } \\
(20-36 \text { years }) \\
(n=1522)\end{array}$} & \multicolumn{3}{|c|}{$\begin{array}{l}\text { Wheeze or } \\
\text { asthma (36 years) } \\
(n=2495)\end{array}$} & \multicolumn{3}{|c|}{$\begin{array}{l}\text { Mean height } \\
\text { adjusted PEFR } \\
\text { at } 36 \text { years } \\
(n=2075)\end{array}$} \\
\hline & & $\%$ & $\begin{array}{l}\text { Test } \\
\text { statistic }\end{array}$ & $p$ & $\%$ & $\begin{array}{l}\text { Test } \\
\text { statistic }\end{array}$ & $p$ & $\%$ & $\begin{array}{l}\text { Test } \\
\text { statistic }\end{array}$ & $p$ & $\begin{array}{l}\text { Mean } \\
P E F R\end{array}$ & $\begin{array}{l}\text { Test } \\
\text { statistic }\end{array}$ & $p$ \\
\hline $\begin{array}{l}\text { Asthma history } \\
\text { (up to } 36 \text { years) }\end{array}$ & $\begin{array}{l}\text { No } \\
\text { Yes }\end{array}$ & $\begin{array}{r}7 \\
17\end{array}$ & 10.554 & $<0.01$ & $\begin{array}{l}23 \\
57\end{array}$ & $47 \cdot 62$ & $<0.001$ & & & & $\begin{array}{l}482 \cdot 9 \\
447 \cdot 4\end{array}$ & 17.97 & $<0.001$ \\
\hline $\begin{array}{l}\text { Cigarette } \\
\text { smoking at } \\
36 \text { years) }\end{array}$ & 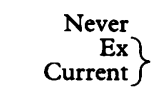 & $\begin{array}{r}8 \\
15\end{array}$ & $8 \cdot 879^{a}$ & $<0.01$ & $\begin{array}{l}31 \\
47\end{array}$ & $23 \cdot 86^{\mathrm{a}}$ & $<0.001$ & $\begin{array}{l}17 \\
33\end{array}$ & $61 \cdot 034^{\mathrm{a}}$ & $<0.001$ & $\begin{array}{l}467 \cdot 7 \\
468 \cdot 6 \\
459 \cdot 1\end{array}$ & $3 \cdot 68$ & $<0.05$ \\
\hline $\begin{array}{l}\text { Chest illness } \\
\text { before } 2 \text { years }\end{array}$ & $\begin{array}{l}\text { No } \\
\text { Yes }\end{array}$ & $\begin{array}{r}9 \\
14\end{array}$ & $6 \cdot 818$ & $<0.01$ & $\begin{array}{l}34 \\
43\end{array}$ & $7 \cdot 45$ & $<0.01$ & $\begin{array}{l}21 \\
27\end{array}$ & $9 \cdot 489$ & $<0.01$ & N/A & & \\
\hline $\begin{array}{l}\text { Atmospheric pollution } \\
\text { exposure } 2-11 \text { years }\end{array}$ & $\begin{array}{l}\text { Low } \\
\text { High }\end{array}$ & N/A & & & $\begin{array}{l}35 \\
43\end{array}$ & $6 \cdot 81$ & $<0.01$ & $\begin{array}{l}23 \\
26\end{array}$ & $3 \cdot 1$ & NS & N/A & & \\
\hline $\begin{array}{l}\text { Parental history } \\
\text { of bronchitis }\end{array}$ & $\begin{array}{l}\text { No } \\
\text { Yes }\end{array}$ & $\begin{array}{r}9 \\
14\end{array}$ & $4 \cdot 307$ & $<0.05$ & $\begin{array}{l}35 \\
43\end{array}$ & 6.99 & $<0.01$ & $\begin{array}{l}19 \\
30\end{array}$ & $29 \cdot 109$ & $<0.001$ & $\begin{array}{l}468 \cdot 8 \\
461 \cdot 5\end{array}$ & $4 \cdot 02$ & $<0.05$ \\
\hline $\begin{array}{l}\text { Crowding at age } \\
2 \text { years (persons } \\
\text { per room) }\end{array}$ & $\begin{array}{l}<1 / \text { room } \\
>1 / \text { room }\end{array}$ & $\begin{array}{r}9 \\
14\end{array}$ & $5 \cdot 62$ & $<0.05$ & N/A & & & $\begin{array}{l}20 \\
28\end{array}$ & $20 \cdot 307$ & $<0.001$ & $\begin{array}{l}471 \cdot 5 \\
458 \cdot 8\end{array}$ & 14.90 & $<0.001$ \\
\hline $\begin{array}{l}\text { Social class } \\
\text { in childhood }\end{array}$ & $\begin{array}{r}\text { Non-manual } \\
\text { Manual }\end{array}$ & $\mathrm{N} / \mathrm{A}$ & & & N/A & & & $\mathbf{N} / \mathbf{A}$ & & & $\begin{array}{l}467 \cdot 7 \\
462 \cdot 6\end{array}$ & $2 \cdot 34$ & NS \\
\hline
\end{tabular}

PEFR = peak expiratory flow rate; $\mathrm{N} / \mathrm{A}=$ not significant in table III or IV and not included in this analysis

${ }^{a}$ Current smoking $v$ never smoked + ex-smokers together

atmospheric pollution between two and 11 years remained associated with adult lower respiratory illness.

The attributable risk of childhood and later factors was calculated for each adult lower respiratory outcome (table VII). In childhood the factors responsible for the greatest amount of excess risk of all lower respiratory problems were a combination of low social class, overcrowding at two years, and lack of amenities. Smoking gave the highest attributable risk of later factors for all lower respiratory outcomes, although the risk of low PEFR attributable to smoking was small compared to that of other symptoms. The risk attributable to childhood factors was greater than that attributable to later experience for all outcomes, except phlegm.

OFFSPRING

Chest illness in first born offspring

Among the cohort's first born offspring, 12.2\% were reported to have had bronchitis, pneumonia, or bronchopneumonia by two years (compared with $25 \%$ of their parents by this age), and eight of

Table VII Attributable risk of associated factors in four indices of adult respiratory health

\begin{tabular}{|c|c|c|c|c|}
\hline & $\begin{array}{l}\text { Phlegm at } \\
25 \text { and } \\
36 \text { years } \\
(n=1348)\end{array}$ & $\begin{array}{l}\text { Adult lower } \\
\text { respiratory } \\
\text { illness at } \\
20-36 \text { years } \\
(n=1353)\end{array}$ & $\begin{array}{l}\text { Wheeze } \\
\text { and asthma } \\
\text { at } 36 \text { years } \\
(n=2086)\end{array}$ & $\begin{array}{l}P E F R^{\star} \\
\text { at } \\
36 \text { years } \\
(n=1330)\end{array}$ \\
\hline $\begin{array}{l}\text { Lower respiratory illness } \\
\text { Parental bronchitis } \\
\text { Poor home circumstances } \\
\text { (overcrowding, manual class, } \\
\text { lacking amenities) } \\
\text { Pollution (0-2 years) }\end{array}$ & $\begin{array}{l}\text { Childhc } \\
16.5 \% \\
21.9 \%\end{array}$ & $\begin{array}{l}\text { risk factors } \\
11 \cdot 5 \% \\
9 \cdot 7 \% \\
\\
17 \cdot 4 \% \\
8 \cdot 6 \%\end{array}$ & $\begin{array}{l}10 \cdot 7 \% \\
11.5 \%\end{array}$ & $\begin{array}{l}\mathbf{2 . 9} \% \\
\mathbf{6 . 8} \%\end{array}$ \\
\hline $\begin{array}{l}\text { Smoking (ever/never) } \\
\text { Asthma } \\
\text { Pollution (2-11 years) }\end{array}$ & $\begin{array}{l}\text { Later } \\
41 \cdot 5 \% \\
7 \cdot 7 \% \\
5 \cdot 5 \%\end{array}$ & $\begin{array}{l}k \text { factors } \\
30 \cdot 7 \% \\
9.6 \% \\
11 \%\end{array}$ & $\begin{array}{r}39 \cdot 7 \% \\
8 \cdot 8 \%\end{array}$ & $\begin{array}{l}7 \cdot 6 \% \\
4 \cdot 1 \% \\
4 \cdot 3 \%\end{array}$ \\
\hline $\begin{array}{l}\text { Childhood risk factors } \\
\text { adjusted for adult factors } \\
\text { Adult risk factors } \\
\text { adjusted for childhood } \\
\text { factors }\end{array}$ & $\begin{array}{l}\text { Adjusted } \\
28 \cdot 3 \%\end{array}$ & $\begin{array}{l}\text { ributable risk } \\
35 \cdot 5 \%\end{array}$ & $57 \cdot 4 \%$ & $28.5 \%$ \\
\hline
\end{tabular}

*Lowest quartile of height and sex adjusted PEFR compared with the other three quartiles these 1654 children also had asthma. In addition a further 22 children $(1.3 \%)$ were reported to have had asthma by two years, apparently a considerable increase in the prevalence of asthma, but a difference subject to difficulties of interpretation. ${ }^{29}{ }^{30}$ The total prevalence of these four conditions by two years was $13 \cdot 6 \%$.

There were no significant differences either in rates of contact between those who had had early life lower respiratory illness and those who had not, or between those from non-manual and manual class families of origin, suggesting that reduction in the prevalence of lower respiratory illness among the offspring was not due to reduced follow up of certain groups.

Although the proportion of children in manual class families was much smaller in the offspring generation they were still at greater risk of lower respiratory illness $(17 \cdot 1 \%)$ than their age peers in non-manual families $(11.6 \%)(p<0.01)$.

Children of parents who had had lower respiratory illness were themselves at greater risk than others of lower respiratory illness before two years $(p<0.001)$, and also had increased risk of early childhood lower respiratory illness if both parents and grandparents had experienced such illness $(p<0.01)$ (analyses not shown). There were no associations between housing circumstances, home ownership, mothers' education, or intergenerational rise in social class and lower respiratory illness in the offspring generation.

The risk of lower respiratory illness by age two years among offspring was greatest in those whose study member parent had experienced early childhood lower respiratory illness and had been a smoker $(16 \%$ of offspring had lower respiratory illness), and less among those whose parents had experienced early life lower respiratory illness but not been smokers $(13 \%)$, or had been smokers but had not had early life lower respiratory illness $(10 \%)$. The risk was least in those whose parents had not experienced lower respiratory illness in childhood and who had never smoked $(8 \%)$.

After adjusting for cohort member parent's smoking, adult social class, and lower respiratory problems in early life and adulthood, only the 
effects on offsprings' lower respiratory illness of their parent's early life lower respiratory disease (test statistic $=5.32$ with $1 \mathrm{df}, \mathrm{p}<0.05$ ) and their parent's experience of adult lower respiratory problems (test statistic 5.03 with $1 \mathrm{df}, \mathrm{p}<0.05$ ) remained significant.

\section{Discussion}

This study shows the accumulation of risk factors for lower respiratory illness within the lifetime of a population born in 1946, and the relative importance of childhood factors.

In childhood the combination of poor home circumstances, atmospheric pollution, and parental chest illness presented a significantly increased risk to child respiratory health: a quarter of children in this study had lower respiratory illness by age two years. The independent effect of poor home circumstances in childhood may be partly attributable to poor nutrition which is likely in such households, particularly in the period when the study population was aged under two years $(1946-48)$. In such circumstances breast feeding may offer protection against chest illness. ${ }^{16}$ Poor home circumstances may have been associated with delay in seeking medical care in these years before the National Health Service began in 1948, and may also have been associated with a greater risk of infection and its exacerbation by damp, cold, and unhygienic surroundings, the extremes of which were considerably greater than today. ${ }^{31}$

The influence of parental lower respiratory illness as a risk factor of childhood lower respiratory tract disease may be explained by cross infection, by family predisposition to lower respiratory disease, ${ }^{32}$ and to some extent by smoking. Although there is no information in this study on smoking habits of study members' parents, the first two years of the study members' lives (194648) were a time of high smoking rates, ${ }^{33}$ and the evident importance of parental smoking as a risk factor for lower respiratory illness in children has been shown in other studies, ${ }^{20-22}$ and in this study was a significant risk factor among the offspring of cohort members.

In adult life the experience of lower respiratory illness before two years, and the risk factors associated with it, particularly poor social circumstances, were independent sources of risk for adult lower respiratory problems and poor PEFR. The fact that poor social circumstances in early life were an independent source of risk for adult lower respiratory problems suggests that some aspect of development may be implicated. Smoking during adolescence and adulthood was a further independent risk factor.

By the time first children were born to study members, better home environment and nutrition had improved child health, as shown, for example, by the secular trend in height gain, ${ }^{34}$ and by the reduction of almost a half in prevalence of lower respiratory disease between birth and age two years. In the offspring generation, however, children from manual social class families were again at greater risk of lower respiratory illness before two years. Early childhood lower respiratory illness was significantly associated with chest illness in parents, with parental smoking, and possibly also with the relatively poor nutrition and home circumstances, concentrated in this now proportionately smaller group. ${ }^{35}$

Comparison of the two generations suggests that reduction in poor home circumstances of children, in atmospheric pollution, and in parental smoking have helped significantly in the reduction of prevalence of lower respiratory illness before the age of two years. This, in turn, should help to reduce the risk of lower respiratory problems in the adult life of the offspring generation.

Reduction in smoking among young people would be likely to bring further significant improvement in subsequent adult respiratory health, reduced risk of impaired respiratory function, and consequent gain in the respiratory health of offspring. However current trends suggest that relatively little change in prevalence of smoking is to be expected in the near future in later born cohorts. ${ }^{36}{ }^{37}$ The evident accumulation during childhood and early adult life of risk of subsequent adult lower respiratory problems suggests that reduction in such problems in the population is likely to take a long time, as each generation carries its accumulated risk forward into middle life and old age.

As members of the 1946 birth cohort grow older we hope to see who will have lower respiratory illness for the first time in later middle life or old age, and whether those hypothetically at risk, but apparently not yet ill with chest disease by 36 years, have a significantly greater risk than others of such relatively late first onset lower respiratory illness. There is some indication of a higher risk of respiratory problems in those so far free from lower respiratory illness, but who had experienced early childhood risk, in their significant overrepresentation among those with poor PEFR at 36 years.

1 Reid DD. The beginnings of chronic bronchitis. Proc $R$ Soc Med 1969; 62: 311-6.

2 Holland WW, Halil T, Bennett AE, Elliott A. Factors influencing the onset of chronic respiratory disease. $B M \mathcal{F}$ 1969; ii: $205-8$.

3 Colley JRT, Reid DD. Urban and social origins of childhood bronchitis in England and Wales. BMF 1970; ii: 213-7.

4 Bland JM, Holland WW, Elliott A. The development of respiratory symptoms in a cohort of Kent schoolchildren. Bull Physiopathol Respir 1974; 10: 699-716.

5 Holland WW, Bailey P, Bland JM. Long term consequences of respiratory disease in infancy. $\mathcal{F}$ Epidemiol Community Health 1978; 32: 256-9.

6 Mok JYQ, Simpson H. Outcome for acute bronchitis, bronchiolitis and pneumonia in infancy. Arch Dis Child 1984; 59: 306-9.

7 Douglas JWB, Waller RE. Air pollution and respiratory infection in children. $\mathrm{Br} \mathcal{F}$ Prev Soc Med 1966; 20: 1-8.

8 Colley JRT, Douglas JWB, Reid DD. Respiratory disease in young adults: influences of early childhood lower respiratory tract illness, social class, air pollution and smoking. $B M \mathcal{F}$ 1973; ii: 195-8.

9 Kiernan KE, Colley JRT, Douglas JWB, Reid DD. Chronic cough in young adults in relation to smoking habits, childhood environment and chest illness. Respiration 1976; 33: $236-44$.

10 Strachan DP, Anderson HR, Bland JM, Peckham C. Asthma as a link between chest illness in childhood and Asthma as a link between chest illness in childhood and
chronic cough and phlegm in young adults. $B M \mathcal{1} 1988 ; 296$ : 890-3.

11 Cooreman J, Reden S, Levallois M, Liard R, Perdrizet S Respiratory history during infancy and childhood, and respiratory conditions in adulthood. Int $\mathcal{f}$ Epidemiol 1990 19: $621-7$

Britten N, Davies JMC, Colley JRT. Early respiratory experience and subsequent cough and peak expiratory flow rate in 36 year old men and women. $B M \mathcal{F} 1987 ; 294$

3 Day MP, Tucker JS, Florey C du V, Ogston S, Mason CM, Donnelly JD. The Mackenzie Institute Record Studymethods and preliminary findings. Health Bull 1989; 47 
14 Burrows B, Knudson RJ, Lebowitz MD. The relationship of childhood respiratory illness to adult obstructive airway disease. Am Rev Respir Dis 1977; 115: 751-60.

15 Reid DD, Fletcher CM. International studies in chronic respiratory disease. Br Med Bull 1971; 27: 59-64.

16 Barker DJP, Osmond C. Child respiratory infection and Barker DJP, Osmond C. Child respiratory infection and
adult chronic bronchitis in England and Wales. BMF 1986;
293: 1271-5.

17 Anderson HR. Respiratory disease in childhood. $\mathrm{Br}$ Med Bull 1986; 42: 361-8.

18 Barker DJP, Osmond C, Carr CM. The intrauterine and early postnatal origins of cardiovascular disease and chronic bronchitis. F Epidemiol Community Health 1989; 43 $237-40$.

19 McCarthy P, Byrne D, Harrisson S, Keithley J. Respiratory conditions: effects of housing and other factors. $\mathcal{F}$ Epidemiol Community Health 1985; 39: 15-9.

20 Colley JRT. Respiratory symptoms in children and parental smoking and phlegm production. $B M \mathcal{F} 1974$; ii: $201-4$.

21 Tager IB, Weiss ST, Munoz A, Rosner B, Speizer FE. Longitudinal study of the effects of maternal smoking on pulmonary function in children. $N$ Eng $₹$ Med 1983; 309: 699-703.

22 Burr ML, Miskelly FG, Butland BK, Merreth TG, Vaughan-Williams E. Environmental factors and symptoms in infants at high risk of

23 Melia RJW, Florey C du V, Chinn S. Respiratory illness in British schoolchildren and atmospheric smoke and sulphur dioxide 1973-7. I Epidemiol Community Health 1981; 35: dioxide 13.

24 Watkins CJ, Leeder SR, Corkhill RT. The relationship between breast and bottle feeding and respiratory illness in between breast and bottle feeding and respiratory illness in
the first year of life. $\mathcal{F}$ Epidemiol Community Health 1979; the first year $180-2$.
33:
25 Wadsworth MEJ. Follow-up of the first national birth cohort: findings from the Medical Research Council National Survey of Health and Development. Paediatr Perinat Epidemiol 1987; 1: 95-117.

26 MRC Committee on the Aetiology of Chronic Bronchitis. Standardised questionnaires on respiratory symptoms. BMF 1960; ii: 1665 .

27 Wadsworth MEJ. Inter-generational differences in child health. In: Measuring socio-demographic change. OPCS health. In: Measuring socio-demographic change. OPCS

28 Wadsworth MEJ. The imprint of time: childhood, history and adult life. Oxford: Oxford University Press, 1991.

29 Burr ML, Butland BK, King S, Vaughan-Williams E. Burr ML, Butland BK, King S, Vaughan-Williams E.

30 Anonymous. Asthma (Commentary). f Epidemio Community Health 1990; 44: 177-8.

31 Joint Committee of the Royal College of Obstetricians and Gynaecologists and the Population Investigation Committee. Maternity in Great Britain. Oxford: Oxford University Press, 1948.

32 Anderson HR, Bland JM, Patel S, Peckham C. The natural history of asthma in childhood. $\mathcal{f}$ Epidemiol Community Health 1986; 40: 121-9.

33 Wald N, Kiryluk S, Darby S, Doll R, Pike M, Peto R, eds. U.K. smoking statistics. Oxford: Oxford University Press, 1988

34 Kuh D, Wadsworth MEJ. Parental height: childhood environment and subsequent adult height in a national birth cohort. Int 7 Epidemiol 1989; 18: 663-8.

35 Whitehead $M$. The health divide. Health Education Council Penguin Books, 1987.

36 General Household Survey 1986. London: HMSO, 1986.

37 Office of Population Censuses and Surveys. Cigarett Office of Population Censuses and Surveys. Cigarette
smoking 1972 to 1988. London: OPCS Monitor, 1989.

Mr fohn Rayner, a medical officer of the Stockport union, gives the following account of the lodging-houses in that town:-

"The lodging-houses in these districts, which are principally occupied by the Irish labourers, are for the most part very much crowded, and are in a remarkably filthy state. The beds and bedding are not only loathsome to the sight but are extremely offensive to the smell, and are so closely packed that several families may occupy the same room, each bed containing several persons. In such places the married and single often repose together, and the beds are so arranged, that in some instances there is not room for a person to walk between them. I have seen seven persons in the same bed, and last week removed to the infirmary a case of rheumatic fever, with translation of the disease to the heart, from a bed which every night contains eight persons. I have generally found that the lower order of Irish labourers occupy the most filthy districts, and that wherever they colonize, misery and wretchedness is sure to abound. They are the most common applicants for medical relief at our charity."

"I lately had a case of inflammation of the absorbments of the legs, from a trifling injury to the foot, in an Irish boy, who was living in a dark, damp cellar, about four yards square, in which were two beds. The height of the ceiling was not more than six feet, and yet seven persons laid in it, together with a few rabbits. One of the beds had to be removed from the wall on account of its extreme dampness, and so dark was the dwelling at mid-day, that I had to make use of a candle whilst inspecting him."

Report to Her Majesty's Principal Secretary of State for the Home Department, from the Poor Law Commissioners on an inquiry into the Sanitary Condition of the labouring population of Great Britain, 1842 